\title{
Properties of Aqueous Mixtures of Pure Salts. Thermodynamics of the Ternary System: Water-Sodium Chloride-Calcium Chloride at $25{ }^{\circ} \mathrm{C}$
}

\author{
R. A. Robinson and V. E. Bower
}

Institute for Materials Research, National Bureau of Standards, Washington, D.C.

(April 20, 1966)

\begin{abstract}
Isopiestic vapor pressure measurements have been made to determine some thermodynamic properties of the system: water-sodium chloride-calcium chloride at $25{ }^{\circ} \mathrm{C}$. Equations are derived for the activity coefficient of each salt in the presence of the other when the total ionic strength is kept constant. The limiting conditions in very dilute solutions are considered and "trace" activity coefficients calculated. A comparison with previous work is made. The excess free energy of mixing is calculated.
\end{abstract}

Key Words: Calcium chloride, isopiestic measurements, mixed salt solutions, sodium chloride, vapor pressure of aqueous solutions.

\section{Introduction}

The application of the isopiestic vapor pressure method of determining the activity coefficients of two salts in a mixed salt solution has been described in two previous papers. In one of these $[1]^{1}$ the system water-sodium chloride-barium chloride was described and in the other [2] the system waterpotassium chloride-barium chloride. This paper is concerned with the system water-sodium chloridecalcium chloride.

\section{Definitions}

The definitions and symbols of the previous paper [1] will be retained except that $\mathrm{C}$ now designates calcium chloride.

\section{Experimental Procedure}

The sodium chloride was a portion of that used in earlier work [1]. Calcium chloride was purified in the manner already described [3]; the following impurities were detected by flame photometry: ${ }^{2}$ sodium, $10 \mathrm{ppm}$; potassium, $10 \mathrm{ppm}$; strontium, $300 \mathrm{ppm}$. A stock solution of sodium chloride was made by dissolving a known weight of the salt in a known weight of water. The stock solution of calcium chloride was made by dissolving the salt in water to give a solution approximately $4 m$ in concentration; its exact composition was then determined by coulometric titration. ${ }^{3}$

\footnotetext{
${ }^{1}$ Figures in brackets indicate the literature references at the end of this paper.

2 Analysis by R. W. Burke of the Analysis and Purification Section.

3 Analysis by G. Marinenko of the Analysis and Purification Section.
}

The stock solutions were used to prepare solutions containing both sodium chloride and calcium chloride in known amounts. These solutions were equilibrated in an isopiestic apparatus contained in a thermostat at $25 \pm 0.01{ }^{\circ} \mathrm{C}$. The time of equilibration varied from 3 days for the most concentrated solutions $\left(M_{B} \sim 5.8\right)\left(M_{C} \sim 2.9\right)$ to 7 days for the most dilute solutions $\left(M_{B} \sim 0.5\right)\left(M_{C} \sim 0.4\right)$. After equilibration, the composition of the solutions at equilibrium was determined from the loss or gain of water by each solution.

\section{Isopiestic Data}

The experimental results are given in table 1 . The second column gives the molality $\left(M_{B}\right)$ of the reference solution of sodium chloride. The next two columns give the molalities of sodium chloride $\left(m_{B}\right)$ and of calcium chloride $\left(m_{C}\right)$, respectively, in the mixed solutions. The fifth column gives values of the ionic fraction, $y_{C}$ of calcium chloride in the mixed solution, defined as $y_{C}=1.5 m_{C} / m$, where $m=m_{B}+1.5 m_{C}$. The sixth column gives values of the isopiestic ratio, defined as $R=M_{B} / m$.

Figure 1 is a plot of isopiestic ratio versus the ionic fraction of calcium chloride for some sets of results in table 1. These isopiestic ratios are much higher than those encountered in the sodium chloride-barium chloride system. For example, in the latter system, a solution of $2.7147 \mathrm{~m}$ sodium chloride was found to be in isopiestic equilibrium with a solution of $1.7610 \mathrm{~m}$ barium chloride, $R=1.0277$; we now find that a solution $2.7546 m$ sodium chloride has the same vapor pressure as a solution of $1.5498 \mathrm{~m}$ calcium chloride, 
TABLE 1. Isopiestic ratios for the system water-sodium chloridecalcium chloride at $25{ }^{\circ} \mathrm{C}$

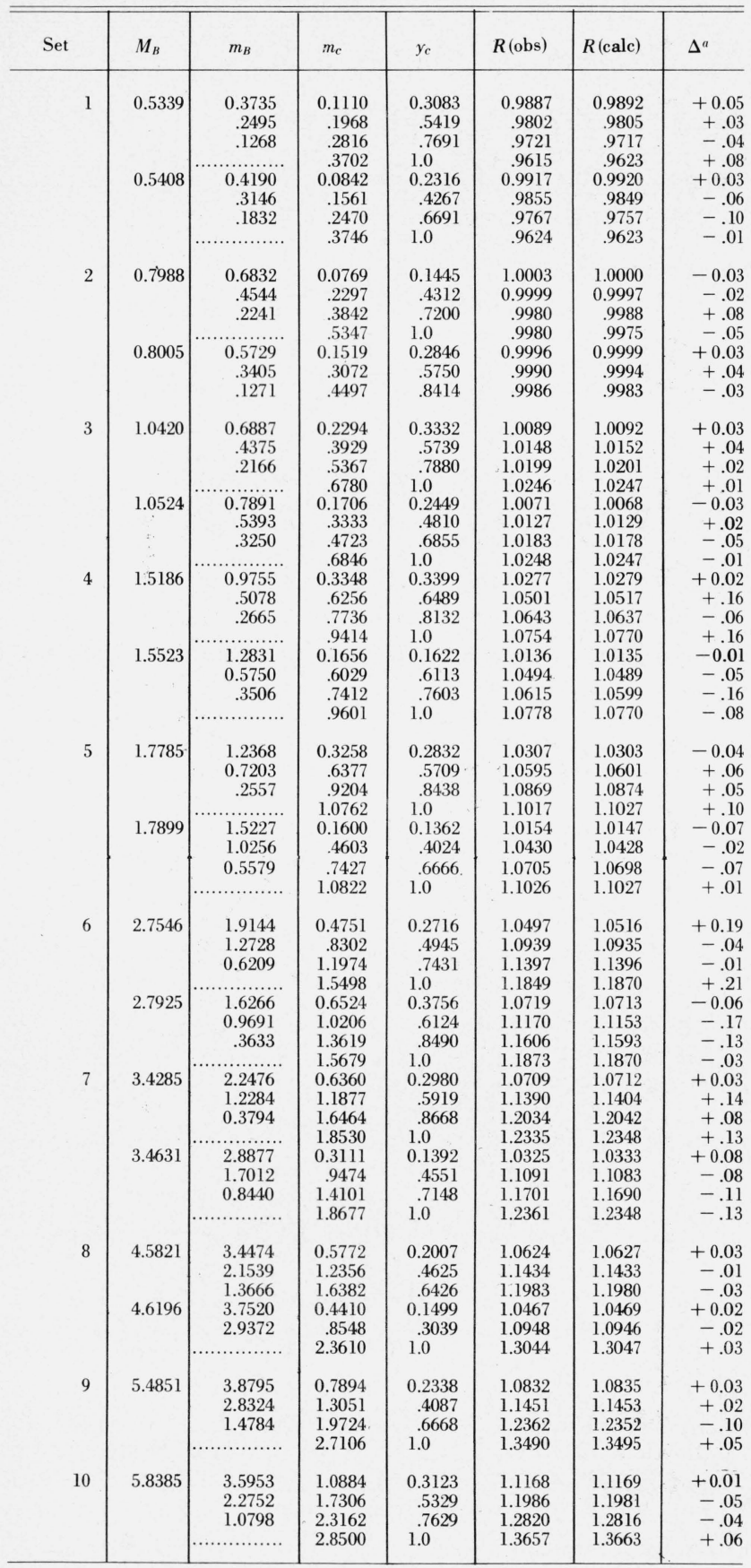

${ }^{a} \Delta=R($ calc $)-R$ (obs), where $R$ (calc) is obtained with the aid of eg (1) and the parameter in table 2 .

$R=1.1849$. Moreover, a plot of isopiestic ratio versus ionic fraction of barium chloride gave a straight line, within experimental error; the plot of isopiestic ratio versus ionic fraction of calcium chloride (fig. 1) may appear to be linear but a closer examination, by the method of least squares, reveals a slight curvature.

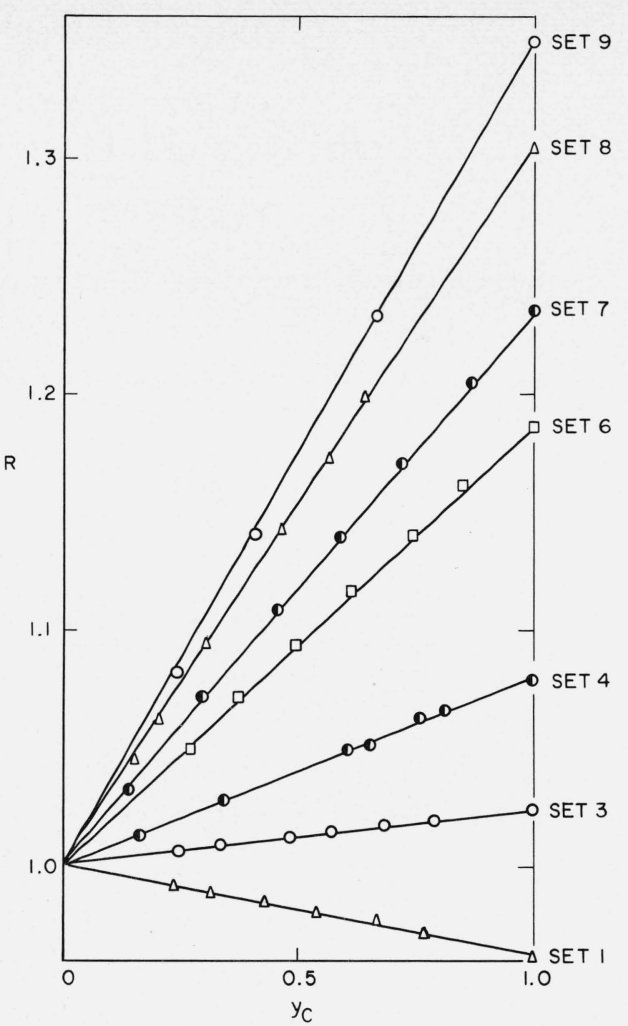

FIGURE 1. Isopiestic ratio of sodium chloride (B)-calcium chloride $(C)$ solutions versus ionic fraction. $R=M_{B} / m, m=m_{B}+1.5 m_{C}, y_{C}=1.5 m_{c} / m$.

The isopiestic results can be represented by the equation

$$
R=M_{B} / m=1-a y_{C}-b y_{C}^{2} .
$$

The parameters of this equation were obtained by the method of least squares. The seventh column of table 1 gives values of $R$ calculated by this equation, using the values of $a$ and $b$ given in table 2 . The last column of table 1 gives the differences between the observed isopiestic ratios and those calculated using eq (1).

TABLE 2. Values of the parameters of eq (1) and of the integral in (5)

\begin{tabular}{r|c|c|r|r|r|r|r}
\hline \hline Set & $\begin{array}{c}M_{B} \\
\text { (mean) }\end{array}$ & $a$ & \multicolumn{1}{c|}{$b$} & \multicolumn{1}{c|}{$b / M_{B}$} & \multicolumn{1}{c|}{$\varphi_{B}$} & \multicolumn{1}{c}{$M_{B} \varphi_{B}$} & \multicolumn{1}{c}{$\int^{a}$} \\
\hline 1 & 0.5374 & +0.0337 & 0.0040 & 0.0074 & 0.9217 & 0.4953 & 0.0015 \\
2 & .7997 & -.0007 & .0032 & .0040 & .9288 & .7428 & .0022 \\
3 & 1.0472 & -.0287 & .0040 & .0038 & .9372 & .9814 & .0026 \\
4 & 1.5355 & -.0828 & .0078 & .0001 & .9583 & 1.4715 & .0038 \\
5 & 1.7842 & -.1087 & .0060 & .0034 & .9715 & 1.7334 & .0041 \\
6 & 2.7736 & -.1909 & .0039 & .0014 & 1.0304 & 2.8579 & .0059 \\
7 & 3.4458 & -.2406 & .0058 & .0017 & 1.0759 & 3.7073 & .0066 \\
8 & 4.6009 & -.3141 & .0094 & .0020 & 1.1609 & 5.3412 & .0074 \\
9 & 5.4851 & -.3595 & .0100 & .0018 & 1.2298 & 6.7456 & .0082 \\
10 & 5.8385 & -.3781 & .0118 & .0020 & 1.2578 & 7.3437 & .0086 \\
\end{tabular}

${ }^{a}$ This column gives values of the integral $0.4343 \int_{0}^{M_{B \varphi}}\left(b / M_{B}\right) d\left(M_{B} \varphi_{B}\right)$. 


\section{Calculation of Activity Coefficients}

The immediate result of an isopiestic experiment is the information that a set of solutions, one of which contains only sodium chloride, another contains only calcium chloride, and the remainder contain both sodium chloride and calcium chloride in different proportions, all have the same vapor pressure and the same water activity, $a_{w}$. It follows that:

$$
2 M_{B} \varphi_{B}=\left(2 m_{B}+3 m_{C}\right) \varphi=3 M_{C} \varphi_{C},
$$

where $\varphi_{B}$ is the osmotic coefficient of the solution containing sodium chloride only at molality $M_{B}, \varphi_{C}$ is the osmotic coefficient of the solution containing calcium chloride only at molality $M_{C}$, and $\varphi$ is the osmotic coefficient of the solution containing both sodium chloride at molality $m_{B}$ and calcium chloride at molality $m_{C}$.

It is the activity coefficients of these two salts in the mixed solution that are required. These are obtained by means of the McKay-Perring equation [4]:

$$
\ln \gamma_{B}=\ln \Gamma_{B}+\ln R+\int_{0}^{M_{B} \varphi_{B}} f\left(m, M_{B}, y_{C}\right) d\left(M_{B} \varphi_{B}\right)
$$

where $\gamma_{B}$ is the activity coefficient of sodium chloride in the mixed solution and $\Gamma_{B}$ that of sodium chloride in the reference solution at molality $M_{B}$. In eq (3),

$$
f\left(m, M_{B}, y_{C}\right)=\frac{1}{m^{2}}\left(\frac{\partial m}{\partial \ln y_{c}}\right)_{a_{w}}+\frac{1}{m}-\frac{1}{M_{B}} .
$$

There are similar equations for $\ln \gamma_{C}$. It can be shown that, if the isopiestic ratio can be represented by eq $(1)$, then the integral term in eq (3) reduces to:

$$
y_{C}^{2} \int_{0}^{M_{B^{\varphi}} \varphi^{2}}\left(b / M_{B}\right) d\left(M_{B} \varphi_{B}\right) .
$$

Figure 2 is a plot of $\left(b / M_{B}\right)$ against $\left(M_{B} \varphi_{B}\right)$. The smooth curve is that of the equation

$$
b / M_{B}=0.0075-0.00243\left(M_{B} \varphi_{B}\right)+0.000234\left(M_{B} \varphi_{B}\right)^{2} .
$$

The integral in eq (3) now becomes:

$$
\begin{aligned}
y_{C}^{2}\left\{0.0075-0.00122\left(M_{B} \varphi_{B}\right)\right. & \\
+ & \left.0.000078\left(M_{B} \varphi_{B}\right)^{2}\right\}\left(M_{B} \varphi_{B}\right)
\end{aligned}
$$

or

$$
\begin{array}{r}
y_{C}^{2}\left\{0.0033-0.00053\left(M_{B} \varphi_{B}\right)\right. \\
\left.+0.00034\left(M_{B} \varphi_{B}\right)^{2}\right\}\left(M_{B} \varphi_{B}\right)
\end{array}
$$

if eq (3) is to be used with decadic logarithms.

There is some scatter of the points in figure 2 because it is not easy to get precise values of the $b$ coefficient, especially from measurements on the more dilute solutions. But the maximum value of the integral that will be used in subsequent calculations is only 0.0060 ; this occurs in the computation of the activity coefficient of calcium chloride in a mixture of total ionic strength $I=4$. Here the logarithm of the isopiestic ratio is -0.0691 and is therefore more important than the integral term. Consequently, we can tolerate some uncertainty about the exact form of the curve in figure 2. For example, had we considered the data best represented by the dashed straight line shown in figure 2 , and carried out the integration of $b / M_{B}$ with respect to $\left(M_{B} \varphi_{B}\right)$ accordingly, the value of the integral would still have been 0.0060 .

The calculation of an activity coefficient can be illustrated by the following example. The mean of two results in set 6 of table 1 shows that a solution of $2.7736 m$ sodium chloride was in isopiestic equilibrium with a solution of $1.5589 \mathrm{~m}$ calcium chloride; the isopiestic ratios of the mixed solutions could be expressed as:

$$
R=1+0.1909 y_{C}-0.0039 y_{C}^{2} .
$$

It has been shown [eq (15) of ref. 2] that the composition of a mixed solution of total ionic strength $I$ in isopiestic equilibrium with a solution of sodium chloride of molality $M_{B}$ can be obtained by solving the quadratic equation:

$$
b I y_{C}^{2}+\left(a I+M_{B}\right) y_{C}=I-M_{B} .
$$

Putting $a=-0.1909, b=0.0039, I=4.5$, we find that $\mathrm{y}_{C}=0.8944$ and hence, by eq $(9), R=1.1676$, log $R=0.0673$. But $m=m_{B}+1.5 m_{C}=M_{B} / R=2.3755$ and therefore $m_{B}=0.2509, m_{C}=1.4164$. At $M_{B}=2.7736$, $\log \Gamma_{B}=-0.1542$ [5]; $\log R$ is 0.0673 and, from eq (8), the integral contributes 0.0052 . Hence, by eq (3) $\log \gamma_{B}=-0.0817$. This is the logarithm of the activity coefficient of sodium chloride in the mixed solution at $I=4.5, y_{C}=0.8944$.

The equation

$$
\log \gamma_{B}=\log \gamma_{B}^{0}-\alpha_{B} I_{C}
$$

is often valid for systems at constant ionic strength.

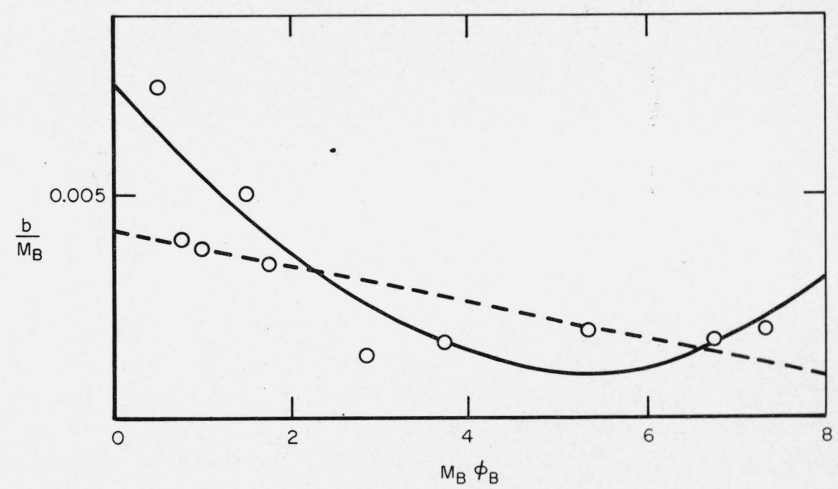

FiguRE 2. b/M $/ M_{B}$ versus $\left(M_{B} \varphi_{B}\right)$. 
Here $\log \gamma_{B}^{0}(=-0.0830)$ [5] is the logarithm of the activity coefficient of sodium chloride in its own solution at a total ionic strength (or molality) of 4.5 and $I_{C}=3 m_{C}=4.2492$. Substituting in eq (11), $\alpha_{B}=-0.0013$.

Similar calculations show that the activity coefficient of calcium chloride in this mixture is given by $\log \gamma_{C}$ $=-0.2065$. But for a solution of calcium chloride only at this total ionic strength $(I=4.5,1.5 \mathrm{~m})$, log $\gamma_{C}^{*}=-0.1222[6]$. Substituting in the equation

$$
\log \gamma_{C}=\log \gamma_{C}^{0}-\alpha_{C} I_{B},
$$

with $I_{B}=m_{B}=0.2509$, gives $\alpha_{C}=-0.0047$.

In this way, values of $\alpha_{B}$ and $\alpha_{C}$ have been calculated at other values of the total ionic strength. They are given in table 3 . The figures in parentheses indicate the set number in table 1 corresponding to the data from which the calculations were made. The values of $\alpha_{B}$ and $\alpha_{C}$ are plotted in figure 3. They lie on a smooth curve except for the values at $I=0.75$ and 1.0; in these cases $\log \gamma_{B}$ and $\log \gamma_{B}^{0}$ are very close together and $\alpha_{B}$ is the result of dividing a small quantity by $I_{C}$, so that it is not surprising that there is some scatter. The same holds for the $\alpha_{C}$ values. "Best" values of $\alpha_{B}$ and $\alpha_{C}$, read from figure 3 , are given in the latter part of table 3 (the significance of the values at $I=0$ in fig. 3 is explained in sec. 6). Table 3 also gives values of $\left(2 \alpha_{B}+\alpha_{C}\right)$. It has been shown that for a system of this valence type $\left(2 \alpha_{B}+\alpha_{C}\right)$ is independent of $I$ if eq (11) and eq (12) are valid, that is to say, no terms in higher powers of $I_{B}$ or $I_{C}$ are needed. Values of $0.5\left(2 \alpha_{B}+\alpha_{C}\right)$ are plotted in figure 3 ; there is a small variation of $\left(2 \alpha_{B}+\alpha_{C}\right)$ with $I$. It could be explained by introducing $\beta_{B} I_{C}^{2}$ and $\beta_{C} I_{B}^{2}$ terms into eq (11) and eq (12), respectively, but $\beta_{B}$ and $\mathrm{B}_{C}$ would be so small that their effect on $\log \gamma_{B}$ and $\log \gamma_{C}$ would be beyond the experimental error.

TABLE 3. Values of $\alpha_{\mathrm{B}}$ and $\alpha_{\mathrm{C}}$ calculated from data in table 1

\begin{tabular}{|c|c|c|c|c|c|}
\hline \multirow{2}{*}{$I$} & \multirow{2}{*}{$\alpha_{B}$} & \multirow{2}{*}{$\alpha_{C}$} & \multicolumn{2}{|c|}{ "Best" values } & \multirow{2}{*}{$\left(2 \alpha_{B}+\alpha_{C}\right)$} \\
\hline & & & $\alpha_{B}$ & $\alpha_{C}$ & \\
\hline 0.75 & $-0.0073(1)$ & 0 & -0.0106 & 0.0005 & -0.0207 \\
\hline 1.0 & $-.0092(2)$ & $0.6002(2)$ & -.0092 & -.0020 & -.0204 \\
\hline 1.5 & $-.0063(3)$ & $-.0098(3)$ & -.0063 & -.0098 & -.0224 \\
\hline 2.0 & $\begin{array}{l}-.0052(4) \\
-.0054(5)\end{array}$ & $\begin{array}{l}-.0130(4) \\
-.0122(5)\end{array}$ & -.0047 & -.0126 & -.0220 \\
\hline 3.0 & $\begin{array}{l}-.0021(5) \\
-.0014(6)\end{array}$ & $\begin{array}{l}-.0165(5) \\
-.0165(6)\end{array}$ & -.0018 & -.0165 & -.0201 \\
\hline 4.0 & $\begin{array}{r}-.0006(6) \\
.0006(7)\end{array}$ & $\begin{array}{l}-.0186(6) \\
-.0185(7)\end{array}$ & 0 & -.0186 & -.0186 \\
\hline 5.0 & $\begin{array}{l}.0007(7) \\
.0006(8)\end{array}$ & $\begin{array}{l}-.0190(7) \\
-.0186(8)\end{array}$ & .0007 & -.0192 & -.0178 \\
\hline 6.0 & $\begin{array}{l}.0016(8) \\
.0024(9)\end{array}$ & $\begin{array}{l}-.0196(8) \\
-.0200(9)\end{array}$ & .0016 & -.0198 & -.0166 \\
\hline 7.0 & $\begin{array}{l}.0014(9) \\
.0014(10)\end{array}$ & $\begin{array}{l}-.0204(9) \\
-.0202(10)\end{array}$ & .0020 & -.0203 & -.0163 \\
\hline
\end{tabular}

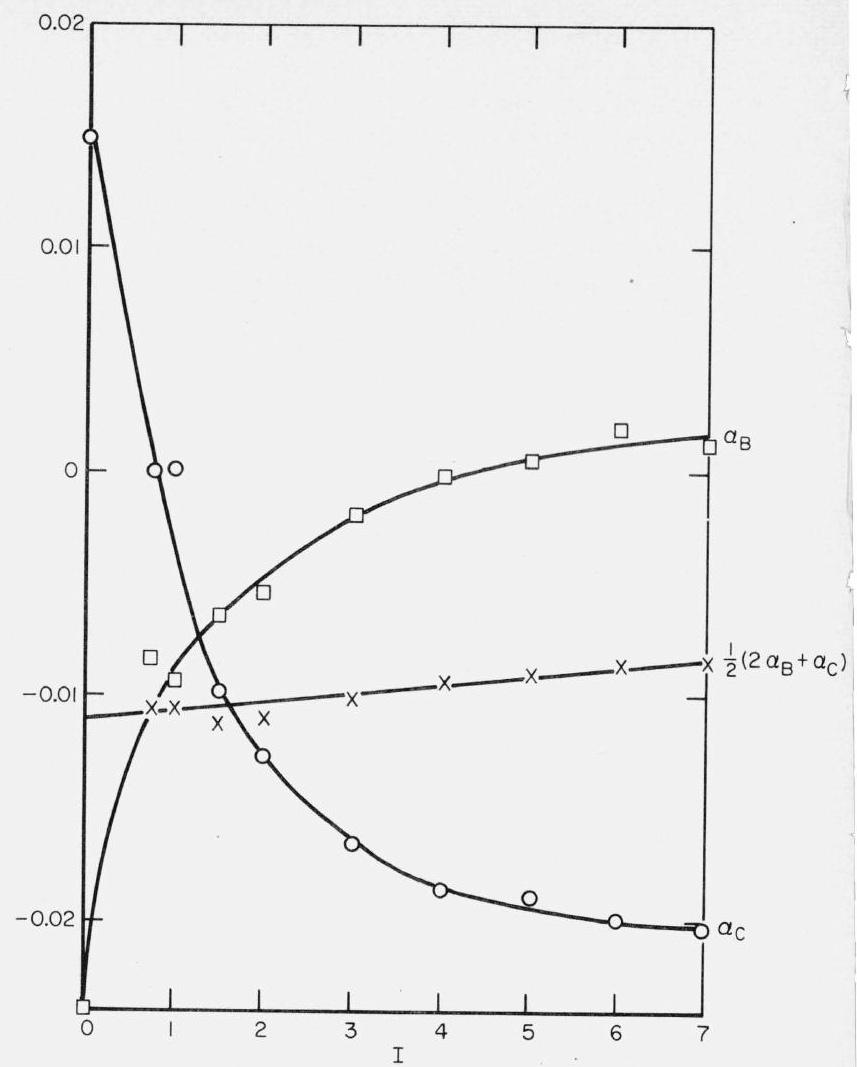

FiguRE 3. $\alpha_{\mathrm{B}}, \alpha_{\mathrm{C}}$, and $0.5\left(2 \alpha_{\mathrm{B}}+\alpha_{\mathrm{C}}\right)$.

\section{Values of $\alpha_{B}$ and $\alpha_{C}$ in Dilute Solutions}

We now consider what values $\alpha_{B}$ and $\alpha_{C}$ might be expected to have when the total ionic strength becomes very small. For this purpose we write for the activity coefficient of the $i$ th ion in a mixed solution $[7,8]$ :

$$
\log \gamma_{i}=\log \gamma^{s t}+\sum_{j} B_{i j} m_{j}
$$

Here $\gamma^{s t}$ is the activity coefficient calculated on the assumption that the only interionic interaction is of the Debye-Hückel type; it can be expressed as

$$
\log \gamma^{s t}=\frac{-z_{i}^{2} A I^{1 / 2}}{1+1.5 I^{1 / 2}}
$$

where $z_{i}$ is the valence of the $i$ th ion. $B_{i j}$ is a coefficient characteristic of the interaction between an $i$ th ion and a $j$ th ion and, in accordance with the Brönsted Principle of Specific Interaction, the summation is to be made over all pairs of oppositely charged ions. Thus, in a solution containing both sodium chloride and calcium chloride,

$$
\log \gamma_{C l}=\log \gamma^{s t}+B_{N a, C l} m_{N a}+B_{C a, C l} m_{C a}
$$




$$
\log \gamma_{N a}=\log \gamma^{s t}+B_{N a, C l} m_{C l}
$$

$\log \gamma_{C a}=4 \log \gamma^{s t}+B_{C a}, C_{C l} m_{C l}$.

Writing $B_{N a, C l}=B_{B}$ and $B_{C a, C l}=B_{C}$ for brevity, and remembering that $2 \log \gamma_{B}=\log \gamma_{N a}+\log \gamma_{C l}$ and $3 \log$ $\gamma_{C}=\log \gamma_{C a}+2 \log \gamma_{C l}$,

$$
\log \gamma_{B}=\log \gamma^{s t}+\left(m_{B}+m_{C}\right) B_{B}+\frac{1}{2} m_{C} B_{C}
$$

and

$\log \gamma_{C}=2 \log \gamma^{s t}+2 / 3 \cdot m_{B} B_{B}+1 / 3 .\left(m_{B}+4 m_{C}\right) B_{C}$.

The activity coefficient of either salt in its own solution in the absence of the other salt, at a total ionic strength $I$, is now given as:

$$
\begin{aligned}
& \log \gamma_{B}^{\circ}=\log \gamma^{s t}+I B_{B} \\
& \log \gamma_{C}^{\circ}=2 \log \gamma^{s t}+4 / 9 . I B_{C}
\end{aligned}
$$

where $I_{B}=m_{B}$ and $I_{C}=3 m_{C}$. These equations enable us to evaluate $B_{B}$ and $B_{C}$ from data on dilute single salt solutions; using the values of Janz and Gordon [9] for sodium chloride and of McLeod and Gordon [10] for calcium chloride, we find $B_{B}=0.007$ and $B_{C}=0.176$.

By $\gamma^{t r}$ we designate the 'trace' activity coefficient of a salt, that is the activity coefficient of a salt when it is present in vanishingly small amount in a solution which contains virtually only the other salt. Equation (18) gives

$$
\log \gamma_{B}^{t r}=\log \gamma^{s t}+1 / 3 . I\left(B_{B}+\frac{1}{2} B_{C}\right)
$$

and eq (19)

$$
\log \gamma_{C}^{t r}=2 \log \gamma^{s t}+1 / 3 . I\left(2 B_{B}+B_{C}\right) .
$$

Hence,

$$
\alpha_{B}=1 / I . \log \gamma_{B}^{\circ} / \gamma_{B}^{t r}=2 / 3 . B_{B}-1 / 6 . B_{C}
$$

and

$$
\alpha_{C}=1 / I . \log \gamma_{C}^{\circ} / \gamma_{C}^{t r}=1.9 \cdot B_{C}-2 / 3 B_{B} .
$$

Substituting numerical values for $B_{B}$ and $B_{C}$, we find $\alpha_{B}=-0.024, \alpha_{C}=0.015$. These values are shown in figure 3 at $I=0$. It will be seen that they are consistent with a reasonable extrapolation of the experimental values at finite concentrations. But $\left(2 \alpha_{B}\right.$ $\left.+\alpha_{C}\right)=-0.0165$, which is in poor agreement with the value of -0.0110 extrapolated from the experimental values. This suggests that the behavior of this system may be more complicated in more dilute solutions.

\section{Trace Activity Coefficients}

In the case when $I_{C} \rightarrow I\left(m_{B} \rightarrow 0\right)$ in eq (11), the trace activity coefficient of sodium chloride is given by

$$
\log \gamma_{B}^{t r}=\log \gamma_{B}^{\circ}-\alpha_{B} I
$$

and similarly

$$
\log \gamma_{C}^{t r}=\log \gamma_{C}^{\circ}-\alpha_{C} I
$$

Using the values of $\alpha_{B}$ and $\alpha_{C}$ in table 3, values of the trace activity coefficients have been calculated. They are shown in figure 4 along with plots of the activity coefficients of each of the salts in its own solution $\left(\gamma_{B}^{\circ}, \gamma_{C}^{\circ}\right)$. This figure is used to emphasize the fact that, at constant total ionic strength, the addition of calcium chloride has only slight effect on the activity coefficient of sodium chloride. On the contrary, addition of sodium chloride has considerable effect on the activity coefficient of calcium chloride.

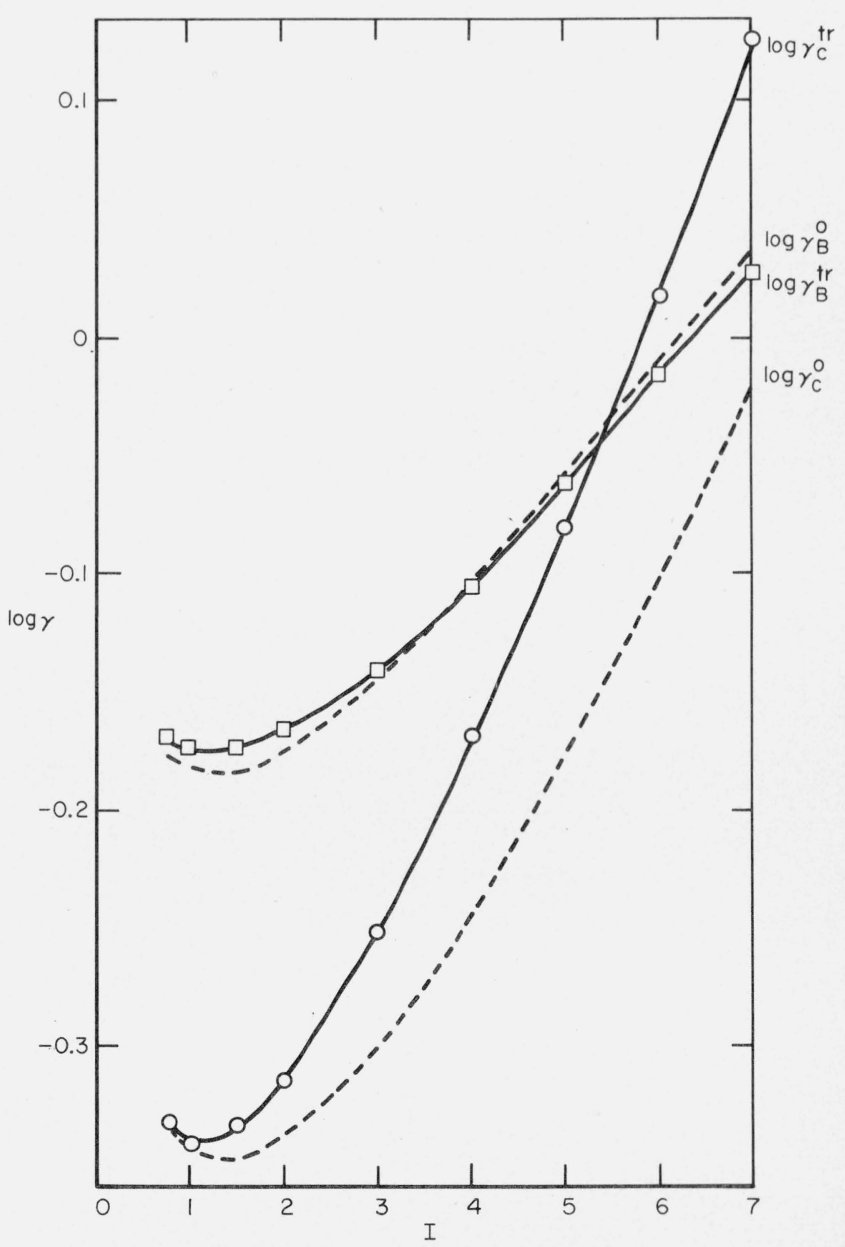

FIGURE 4. Activity coefficients of sodium chloride and calcium chloride.

Activity coefficient of a salt in its own solution. Trace activity coefficient. 


\section{Comparison With Previous Work}

Lanier [11] has used the sodium ion-sensitive glass electrode to measure the activity coefficient of sodium chloride in calcium chloride solutions. In table 4 we compare his values of $\alpha_{B}$ with those obtained in the present work, and also values of $\log \gamma_{B}^{t r}$ calculated therefrom. The agreement is excellent at $I=3.0$ and $I=6.0$. The agreement between the two values of $\alpha_{B}$ at $I=1.0$ is not so good, but the values of $\log$ $\gamma_{B}^{t r}$ are in fair agreement.

TABLE 4. Comparison of $\alpha_{B}$ and $\log \gamma_{\mathrm{R}}^{\mathrm{tr}}$ values

\begin{tabular}{c|r|r|r|r}
\hline \hline \multicolumn{3}{c|}{$\alpha_{B}$} & \multicolumn{2}{c}{$-\log \gamma_{B}^{r r}$} \\
\hline$I$ & This work & Lanier [11] & This work & Lanier \\
\hline 1.0 & -0.0092 & -0.0040 & 0.173 & 0.178 \\
3.0 & -.0018 & -.0020 & .142 & .140 \\
6.0 & .0016 & .0013 & .016 & .014 \\
\hline
\end{tabular}

\section{Excess Free Energy of Mixing}

It has been shown [2] that the excess free energy of mixing $0.5 \mathrm{~kg}$ of water containing 0.5 I moles of a $1: 1$ salt and $0.5 \mathrm{~kg}$ of water containing $0.5(I / 3)$ moles of a 1:2 salt, giving a kilogram of water containing $0.5 I$ moles of the first salt and $0.5(I / 3)$ moles of the other, the total ionic strength of all three solutions being $I$, is given by the expression:

$$
\frac{\Delta G^{E}}{2.303 R T}=-\frac{I^{2}}{8}\left(2 \alpha_{B}+\alpha_{C}\right)
$$

provided that eq (11) and eq (12) hold. Using the values of $\alpha_{B}$ and $\alpha_{C}$ in table 3 , the excess free energy of the sodium chloride-calcium chloride system can be calculated, giving the values shown in table 5 .
TABLE 5. Excess free energy of mixing at $25^{\circ} \mathrm{C}$

\begin{tabular}{c|c|c|c|c|c|c|c|c|c}
\hline \hline$I$ & 0.75 & 1 & 1.5 & 2 & 3 & 4 & 5 & 6 & 7 \\
\hline$\Delta G^{E}$ & 2 & 4 & 9 & 15 & 31 & 51 & 76 & 102 & 136 \\
\hline
\end{tabular}

$\Delta G^{E}$ is expressed in calories per kilogram of water, l calorie $=4.1840 \mathrm{~J}$.

These values of the excess free energy of mixing are opposite in sign but comparable in magnitude with those for the sodium chloride-barium chloride and potassium chloride-barium chloride systems.

Grateful acknowledgment is made to the Office of Saline Water for a grant in partial support of this work.

\section{References}

[1] R. A. Robinson and V. E. Bower, J. Res. NBS 69A (Phys. and Chem.) No. 1, 19 (1965).

[2] R. A. Robinson and V. E. Bower, J. Res. NBS 69A (Phys. and Chem.) No. 5, 439 (1965).

[3] R. A. Robinson and V. E. Bower, J. Res. NBS 70A (Phys. and Chem.) No. 4, 305 (1966).

[4] H. A. C. McKay and J. K. Perring, Trans. Faraday Soc. 49, 163 (1953).

[5] R. A. Robinson and R. H. Stokes, Electrolyte solutions, Appendix 8.3, 2d. ed., (Butterworths Scientific Publications, London, 1959).

[6] R. H. Stokes, Trans. Faraday Soc. 41, 637 (1945); see also ref [5], Appendix 8.5.

[7] E. A. Guggenheim, Phil. Mag. [7] 19, 588 (1935).

[8] G. Scatchard, Chem. Revs. 19, 309 (1936).

[9] G. J. Janz and A. R. Gordon, J. Am. Chem. Soc. 65, 218 (1943).

[10] H. G. McLeod and A. R. Gordon, J. Am. Chem. Soc. 68, 58 (1946).

[11] R. D. Lanier, J. Phys. Chem. 69, 3992 (1965).

(Paper 70A4-406) 